\title{
CORRECTION OF INTENSITY INCIDENCE ANGLE EFFECT IN TERRESTRIAL LASER SCANNING
}

\author{
A. Krooks ${ }^{\text {a, } * \text {, S. Kaasalainen }}{ }^{\text {a }}$, T. Hakala ${ }^{\text {a }}$, O. Nevalainen ${ }^{\text {a }}$ \\ ${ }^{a}$ Department of Photogrammetry and Remote Sensing, Finnish Geodetic Institute, \\ P.O. Box 15, 02431 Masala, Finland - anssi.krooks@fgi.fi
}

KEY WORDS: Terrestrial laser scanning, Laser scanning intensity, incidence angle effect,

\begin{abstract}
:
In this article, we have studied the incidence angle effect on Terrestrial Laser Scanning (TLS) intensity. In previous tests, it has been found that the backscattered intensity of an object affects the incidence angle effect. We made additional experiments to investigate the potential mixing of distance and incidence angle effects and the role of surface parameters such as object grain size and scanning wavelength. The results indicate that distance and incidence angle effects do not mix and laboratory measured correction values can be used to correct intensity data from field-scanned point clouds. We also compared the laboratory measurements to real world surfaces to validate the correction procedures in practical TLS applications. The idea is also to make practical recommendations for TLS intensity correction in most common TLS applications.
\end{abstract}

\section{INTRODUCTION}

The intensity data recorded by airborne and terrestrial laser scanner (TLS) instruments has been studied intensely during the last few years. Radiometric calibration procedures have been suggested and tested for both airborne and terrestrial laser scanning (Coren et al., 2006, Ahokas et al., 2006, Wagner et al., 2006, Höfle et al., 2007 \& Kaasalainen et al., 2009b). The intensity data is mostly applied in the processing and visualization of the range data, and most of the laser scanner studies are still being published without any radiometric calibration of the intensity data. TLS derived intensity information can be used to aid segmentation and classification algorithms alongside geometrical information if the intensity is calibrated. Using external calibration tiepoints, the intensity can also be used in multi-temporal remote sensing. (Höfle et al., 2007 \& Yan et al., 2012).

The backscattered laser signal is affected by the scanning geometry, the surface properties of the target, and atmospheric and instrumental parameters (based on the radar range equation, see, e.g., (Wagner et al., 2006 \& Höfle et al., 2007) for more details and physical basis for the intensity calibration). The scanning geometry is mainly described by two parameters: the range (distance between the scanner and the object) and the incoming beam incidence angle to the target (Kaasalainen et al., 2011, Soudarissanane et al., 2011). The range (distance) effect on intensity depends on several parameters. For TLS, the distance effect seems to depend mostly on the instrument (Kaasalainen et al., 2011 \& Pfeifer et al., 2008), while for ALS the atmospheric effects also play a strong role. The range (distance) effect on TLS intensity is considered to be proportional to $1 / R^{2}$ ( $R$ being the range), but, in practice, a number of instrumental features affect strongly the range vs. distance behavior. The instrumental effects are different for different scanners, so they must be studied individually for each instrument (Pfeifer et al., 2008, Lichti et al., 2003, Voegtle et al., 2009 \& Kaasalainen et al., 2009a).

The incidence angle effect is an object property (related to target scattering properties and surface structure, and also to the scanning geometry) whereas the distance effect is a property of the scanning geometry only. However, it is difficult to link these parameters into laser scanner intensity. Some initial studies exist for TLS (Kaasalainen et al., 2011, Lichti et al., 2005, Pesci et al., 2008 \& Kukko et al., 2008) and ALS (Kaasalainen et al., 2009b \& Lehner et al., 2010), but no correction methods have been established. Another thing that needs further study is the mixing of range and incidence angle effects in TLS data, because instrumental effects are known to affect the measured intensity for most TLS instruments, causing the radar equation not to be applicable at all ranges (Kaasalainen et al., 2011, Pfeifer et al., 2008, Lichti et al., 2003, Kaasalainen et al., 2009a). In many field applications (e.g., those where stationary TLS is carried out with the instrument mounted on a tripod) the incidence angle changes along with the increasing range. The incidence angle effect on ALS intensity has been assumed to be small in many studies (Kaasalainen et al., 2009b, Hollaus et al., 2010), but further studies are called for, especially because it has turned out that the incidence angle does have an effect on data quality and should be taken into account in normalizing ALS data from different flight paths (Jutzi et al., 2009).

We have measured and analyzed the incidence angle effect on TLS intensity in our previous studies (Kaasalainen et al., 2011 \& Kukko et al., 2008). In this article, we present a systematic study of the incidence angle effect for targets with varying grain sizes and reflectance. We have tested the distance and incidence angle correction methods with TLS field data and investigated the possible mixing of these effects in a practical case, where the distance and incidence angle effects almost always occur simultaneously. This is important in the measurement and calibration of TLS and recently increased mobile laser scanner (MLS) intensity, and needs to be taken into account in the intensity correction. We have also extended and optimized the correction procedure introduced in (Kaasalainen et al., 2011 \& Kukko et al., 2008), to find out whether an intensity-based correction of the incidence angle effect would be sufficient in the case of surfaces of intermediate-scale roughness (i.e., excluding the extremes of surface roughness with respect to the laser spot size), or using a reference target with (visually) similar surface properties.

\footnotetext{
* Corresponding author. This is useful to know for communication with the appropriate person in cases with more than one author.
} 


\section{METHODS}

\subsection{Data collection and processing}

The TLS data used in this article was collected with a Leica HDS6100 terrestrial laser scanner (see Kaasalainen et al., 2009a and Table 1 for the scanner specifications). The HDS6100 is a continuous wave TLS and relies on frequency modulation in distance measurements hence the wavelength of the laser beam varies from 650 to 690 nanometers. The resulting point cloud data is processed with the $\mathrm{Z}+\mathrm{F}$ LaserControl 7.4, which allows the raw intensity values to be extracted and also has an optional distance correction to intensity values. Intensity values from different measurements were calibrated by using a 4-step calibration plate with $99 \%, 50 \%, 25 \%$ and $12 \%$ reflectance (Spectralon ${ }^{\circledR}$ ) as a reference. The Spectralon is commonly used in reflectance spectroscopy for reflectance calibration. Since the laser scanner measurement only occurs in backscatter direction only, we call the intensity measured with TLS (and calibrated with the intensity of Spectralon reference measured similarly), i.e., the directional portion of the (hemispherical) reflectance, the backscattered reflectance of the target (cf. Kaasalainen et al., 2009a).

An 8-channel hyperspectral LiDAR system developed at the Finnish Geodetic Institute was also used to scan sand samples to investigate wavelength dependence of the incidence angle effect. This Lidar system produces a 3D point cloud with spectral backscattered reflectance data in 8 spectral channels between 500 and 1000 nanometers, using a supercontinuum laser as a light source (see (Hakala et al., 2012) for more details on the instrument). The wavelength channels in this experiment were $555 \mathrm{~nm}, 624 \mathrm{~nm}, 691 \mathrm{~nm}, 725 \mathrm{~nm}, 760 \mathrm{~nm}, 795 \mathrm{~nm}, 899$ $\mathrm{nm}$, and $1000 \mathrm{~nm}$. For other scanning parameters, see Table 1 .

\begin{tabular}{lcc}
\hline \hline & $\begin{array}{c}\text { Hyperspectral } \\
\text { Lidar }\end{array}$ & Leica HDS6100 \\
\hline Wavelength & $400-1000 \mathrm{~nm}$ & $650-690 \mathrm{~nm}$ \\
$\begin{array}{l}\text { Distance } \\
\text { measurement }\end{array}$ & Time-of-flight & Phase detection \\
$\begin{array}{l}\text { Field-of-view } \\
\begin{array}{l}\text { Beam diameter at } \\
\text { exit } \\
\text { Beam divergence }\end{array}\end{array}$ & $120^{\circ} \times 90^{\circ}$ & $360^{\circ} \times 310^{\circ}$ \\
\hline \hline
\end{tabular}

Table 1. The scanner specifications

The laser beam incidence angle was determined with a built-in feature of the Leica Cyclone 8 software to calculate surface normal vectors. The algorithm fits a plane to 1500 adjacent points of the selected point and calculates the normal vector of that plane. The incident angle was then calculated from plane's normal vector and the point vector between the scanner origin and a measured point on the target surface.

We studied the effect of the following parameters on the incidence angle behavior of TLS intensity. Each experiment has been described in more detail in the following subsections.

In our previous paper (Kaasalainen et al., 2011), we studied the possible mixing of grain size and target reflectance effects. In this study, the aim is to study whether the target reflectance dominates the incidence angle effect and what is the role of target grain size.'

The laser wavelength: As terrestrial laser scanners currently available at the market use wavelengths between 532 and $1064 \mathrm{~nm}$. To study if the laser wavelength affects the incidence angle behavior, we studied the incidence angle effect for some samples using the 8-channel hyperspectral lidar system (Hakala et al., 2010).

Instrumental effects: the measured TLS intensity has been shown to depend strongly on the distance for most TLS instruments (Kaasalainen et al., 2011, Pfeifer et al., 2008 \& Kaasalainen et al., 2009a). In many measurement setups, the distance and incidence angle to the target are varied simultaneously, which causes the intensity vs. distance curve to be affected by both of these parameters (i.e., the incidence angle and the distance to the target). To investigate whether the corrections of these effects are independent of each other in TLS data, we carried out an incidence angle experiment independently at different distances.

We also tested the possible instrumental effects and correction procedures in a 'real-world' case, where both the incidence angle and distance effects were present in the data.

\subsection{The effect of grain size}

We investigated the effect of grain size to the incident angle behavior by scanning a selection of natural sands and white sugar with different grain sizes. We filtered desert sand from Assuan, Egypt, and beach sand from Kivenlahti beach (Espoo, Finland) to five different classes: non-filtered, over $500 \mu \mathrm{m}$, 250-500 $\mu \mathrm{m}, 125-250 \mu \mathrm{m}$, and smaller than $125 \mu \mathrm{m}$. The sugars used were standard commercial products and, according to the manufacturer, with grain sizes of 2500-3500 $\mu \mathrm{m}$ (coarse sugar), 450-600 $\mu \mathrm{m}$ (fine sugar), 350-430 $\mu \mathrm{m}$ (extra fine sugar), and $20-50 \mu \mathrm{m}$ (icing sugar). To study the possible mixed effects of grain size and target intensity, we produced a sample with equal grain size but different intensity by spraying a sample of nonfiltered Kivenlahti sand with matt black paint. The intensity of the painted sand was 0.04 .

The scanner was mounted on top of the sample at distance of 1.65 meters. The sample was tilted using a small goniometer between angles $0^{\circ}$ and $60^{\circ}$. The Spectralon calibration plate was used to calibrate the raw intensity values. Small samples with 3000-4000 points were taken from TLS point clouds and the mean intensity value was calculated from them. Incidence angles greater than $40^{\circ}$ were measured with different geometry, i.e., to achieve incidence angles greater than $0^{\circ}$ on the horizontal sample surface, the sample were not placed directly under the scanner. In this way, we were able to tilt the sample into incidence angles greater than $40^{\circ}$ without the grains falling down because of the tilt.

\subsection{Wavelength effects}

The potential difference in incidence angle effect caused by wavelength dependency was investigated with an active hyperspectral LiDAR developed at the FGI (Hakala et al., 2012). The instrument is a novel type of laser scanner, based on supercontinuum laser technology. The scanner produces a hyperspectral point cloud with the same measurement, i.e., each point in the point cloud comes with an 8-channel reflectance spectrum. This instrument almost covers the wavelength range 
of the commercial TLS instruments currently available on the market. An unfiltered sample of Kivenlahti beach sand and a black painted sample of the same sand were scanned with incidence angles ranging from $0^{\circ}$ to $50^{\circ}$. A goniometer was used to tilt the sample. The mean intensity value at each incidence angle was calculated from 11 measurements containing approximately 5500 points. The results were compared with mean intensities of the same samples scanned with the Leica HDS6100.

\subsection{Simultaneous correction of range and incidence angle effects from TLS data}

Physically, the backscattered signal recorded by a laser scanner is predicted by the radar equation (Wagner et al., 2006) to depend on the target reflectivity and the angle of incidence, whereas the distance is predicted to follow the $1 / R^{2}$ ( $\mathrm{R}$ being the range) dependency for targets larger than the laser footprint (Pfeifer et al., 2008). In practical applications, however, this prediction is not applicable, because there are instrumental modifications (such as brightness reducers at near distances etc.) that are designed to optimize the range measurement. To investigate the potential mixing of range and incident angle effects in TLS intensity measurement and data, we scanned the 4-step calibration plate (Spectralon Inc) at distances of 1, 5 and 10 meters. The plate was tilted from vertical to horizontal orientation with $5^{\circ}$ steps. The distance effect was corrected using a intensity vs. distance function based on data from our previous laboratory measurements (see Kaasalainen et al., 2011 for further information).

Small samples were taken from each panel of the plate with approximately 100000 (distance: $1 \mathrm{~m}), 2000$ (distance: $5 \mathrm{~m}$ ) and 1000 (distance: $10 \mathrm{~m}$ ) points where mean intensity value and surface normal were calculated. We also tested the potential usability of the laboratory measurement as a correction data of the incident angle effect on real world surfaces. We scanned a white wall at distances of 1, 2, 3 and 4 meters. Spectralon reference was used to calibrate the intensity values between individual scans and the distance effect was minimized with correction values from previous laboratory tests.

\subsection{Validation}

To validate the results measured in laboratory with a real world case, we carried out a TLS experiment on the Kivenlahti beach, where some of the laboratory sand samples were taken from. A section of the beach was scanned and incident angle and intensity data were extracted from the point cloud. The Spectralon reference was used to calibrate the intensity to be able to compare the results with laboratory scanned samples of the same sand (see Section C). The distance effect was corrected from the intensity data using automatic function found in the $\mathrm{Z}+\mathrm{F}$ LaserControl software.

\section{RESULTS AND DISCUSSION}

\subsection{The effect of grain size and target reflectance}

Results from the sugar and sand experiment are shown in Fig. 1. We did not find solid dependency between the target grain size and incidence angle effect. The results point out that targets with similar backscattered intensity share a similar trend in the incidence angle behavior and the effect of grain size appears to be minor. However, the grain sizes used in this experiment were considerably smaller than the laser spot on the target and our previous study suggests that variations on the surface topology that are greater than the laser spot have effect on the incidence angle behavior (Kukko et al., 2008).

\subsection{The wavelength effect}

The results from the two measurement series with the hyperspectral lidar, presented in Figs. 3 and 4 indicate that there seems to be no wavelength dependency on the incidence angle effect. Also, the intensity of the sand sample measured with the Leica HDS 6100 is in agreement with the hyperspectral data. This would allow the use of the same incidence angle correction functions for data from different scanners. More experiments with different objects are needed to verify the results, especially when backscattered intensity is high. The reflectance values for sugar samples are between 0.86-0.98, whereas those for Assuan and Kivenlahti samples are around 0.41-0.47 and 0.15-0.29, respectively. The reflectance value for Black Kivenlahti sandsample is around 0.04 . Thus the incidence angle behavior appears to be dominated by target reflectance.

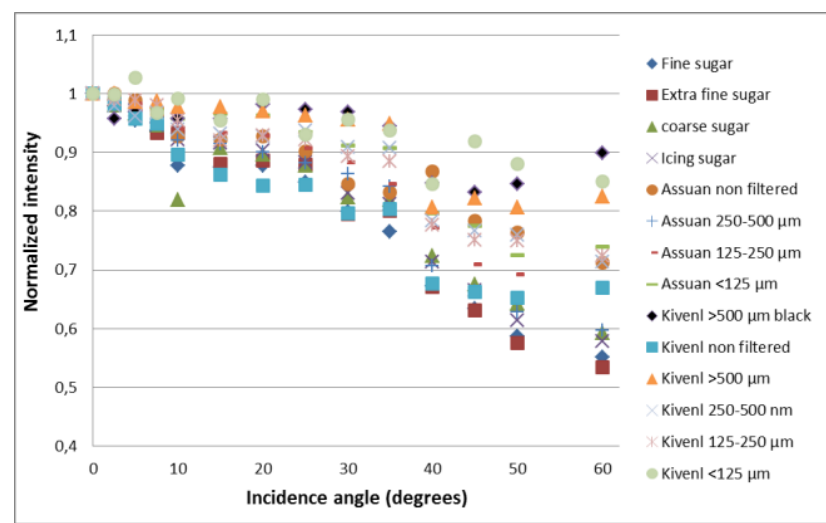

Figure 1. Intensity vs. incidence angle for sand and sugar samples. The intensity is normalized to 1 at $0^{\circ}$. The differences in the incidence angle effect are mostly related to target brightness.

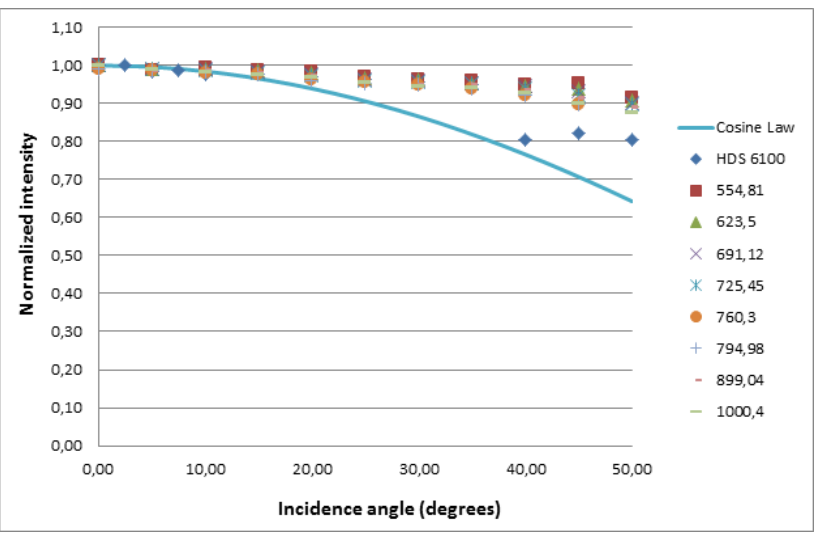

Figure 3. Kivenlahti beach sand (>500 $\mu \mathrm{m}$ grain size) scanned with hyperspectral Lidar, compared with the same sand sample scanned with the Leica HDS6100 from the experiment described in Section 2.3. The wavelength channels are denoted in the legend. Intensity is normalized to 1 at $0^{\circ}$. 


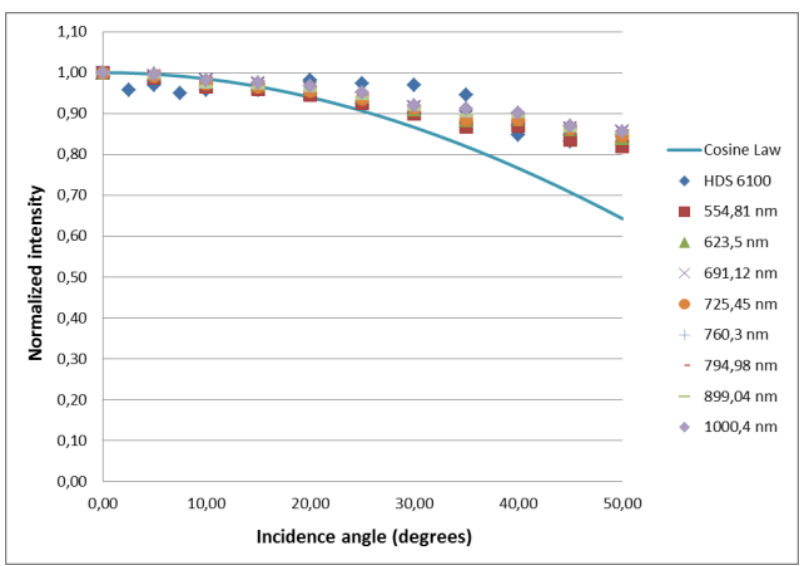

Figure 4. Black-painted Kivenlahti beach sand $(>500 \mu \mathrm{m}$ grain size) scanned with hyperspecral Lidar, compared with the same sand sample scanned with the Leica HDS6100 from the experiment described in section 2.3. The wavelength channels are denoted in the legend. Intensity is normalized to 1 at $0^{\circ}$.

\subsection{Simultaneous correction of range and incidence angle effects}

Results from the incident angle effect experiment for the 4-step Spectralon at different distances are presented in Figs. 5-7. Similar trends for each panel have been observed at different distances $(1 \mathrm{~m}, 5 \mathrm{~m}$, and $10 \mathrm{~m})$. This can also be observed from a combined plot for the 99\% (Fig. 8), 50\% (Fig. 9), 25\% (Fig. 10 ) and $12 \%$ (Fig. 11) panel: after correcting the distance effect from the intensity data, we compared the values of the each Spectralon panels measured at all three distances and found out that the drop in intensity as a function of the incidence angle is similar. This indicates that the measurement of the incidence angle effect is independent of distance, i.e., there seems to be no instrumental effects that would affect its correction from TLS data. It must be noted, however, that the intensity measurement at a close proximity ( 1 meter) of the scanner has been strongly affected by the brightness reducer at near-distances (cf. Kaasalainen et al., 2009a). Nevertheless, similar trends are observed, although there is high noise in the data, which complicates the comparison with results from other distances.

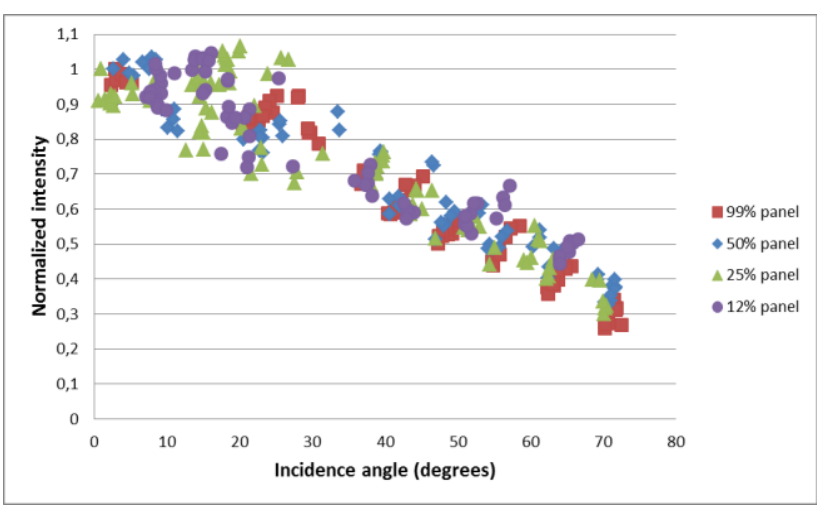

Figure 5. The 4-step Spectralon scanned from $1 \mathrm{~m}$ distance with incident angles ranging from $0^{\circ}$ to $82^{\circ}$. The intensity is normalized to 1 at $0^{0}$

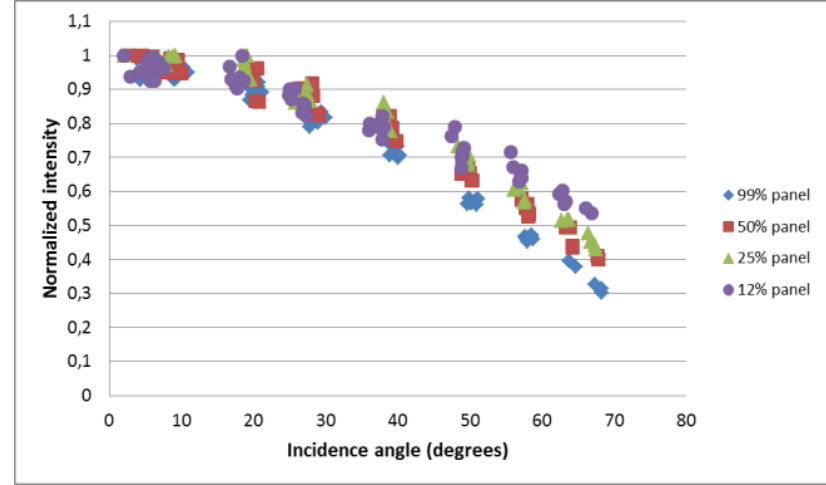

Figure 6. The 4-step Spectralon scanned from $5 \mathrm{~m}$ distance with incident angles ranging from $0^{\circ}$ to $82^{\circ}$

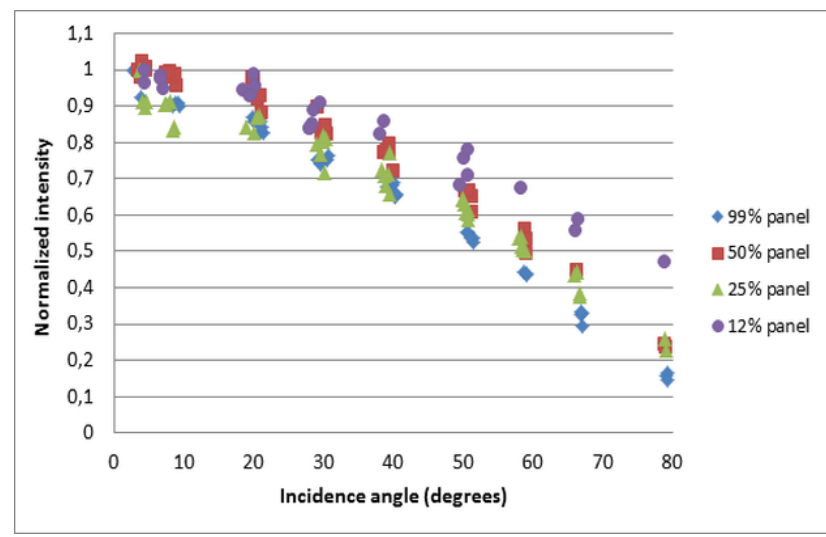

Figure 7. The 4-step Spectralon scanned from $10 \mathrm{~m}$ distance. Incident angles range between $0^{\circ}$ and $80^{\circ}$ with approximately $5^{0}$ steps. The intensity values are normalized to 1 at $0^{0}$.

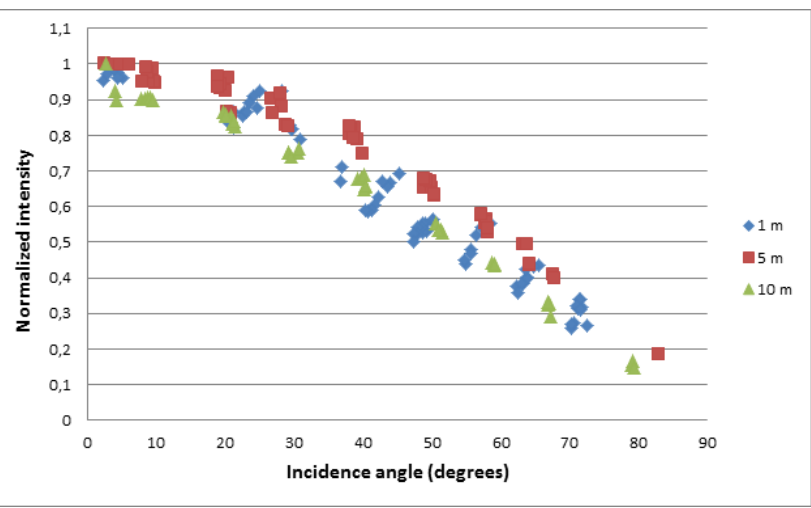

Figure 8. Comparison of the incidence angle effect of the $99 \%$ reflectance Spectralon panel when distance effect has been corrected from the intensity data. Distances of 1,5 and 10 meters are compared. The intensity is normalized to 1 at $0^{\circ}$ 


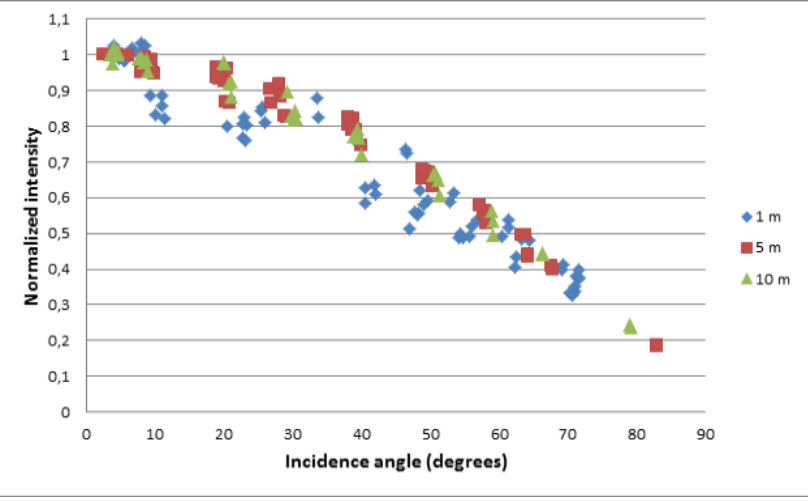

Figure 9. Comparison of the incidence angle effect of the $50 \%$ reflectance Spectralon panel when distance effect has been corrected from the intensity data. Distances of 1,5 and 10 meters are compared. The intensity is normalized to 1 at $0^{0}$

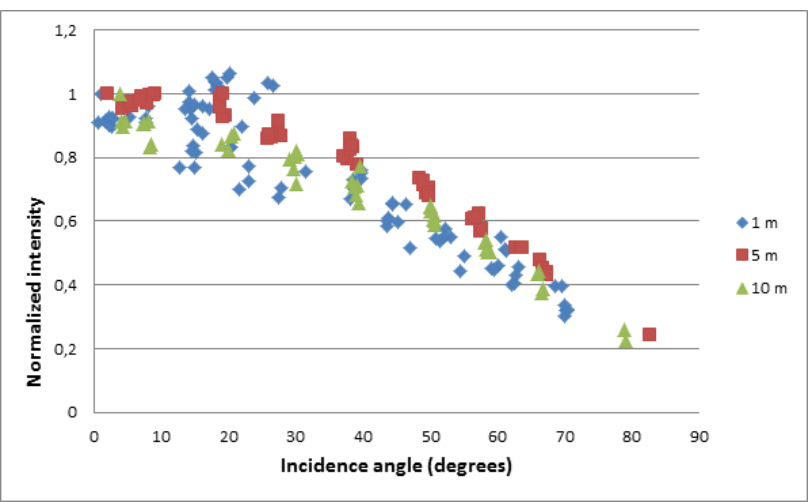

Figure 10. Comparison of the incidence angle effect of the $25 \%$ reflectance Spectralon panel when distance effect has been corrected from the intensity data. Distances of 1,5 and 10 meters are compared. The intensity is normalized to 1 at $0^{0}$

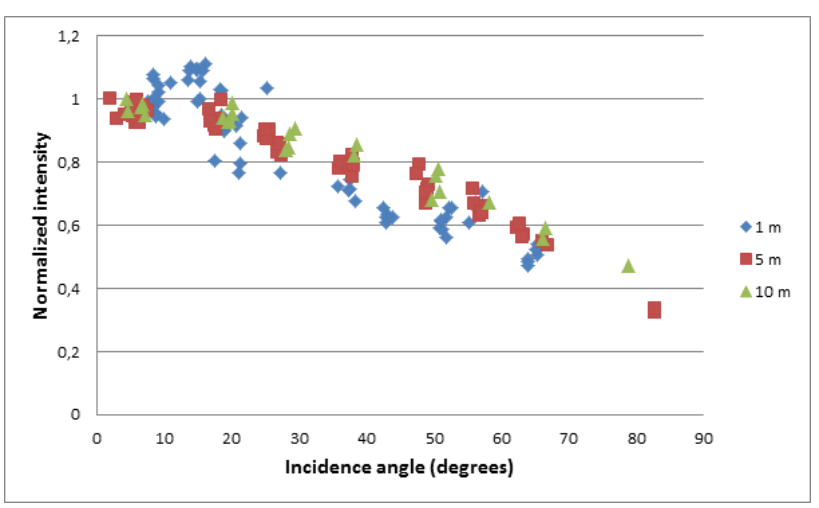

Figure 11. Comparison of the incidence angle effect of the $12 \%$ reflectance Spectralon panel when distance effect has been corrected from the intensity data. Distances of 1,5 and 10 meters are compared. The intensity is normalized to 1 at $0^{0}$

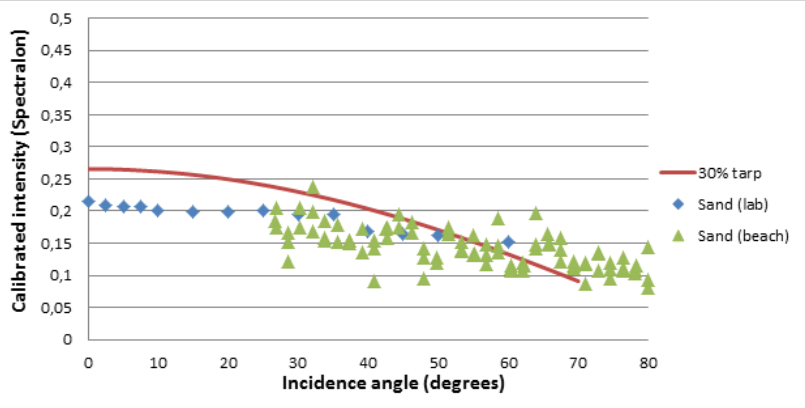

Figure 12. Comparison between Kivenlahti beach sand scanned on the beach and in laboratory and the $30 \%$ reflecting tarp. The intensity has been distance-corrected and calibrated with the Spectralon target.

\subsection{Validation}

The Kivenlahti beach sand scanned in laboratory was compared with intensities extracted from scans made on the actual beach (Fig. 12) when the surface sand was dry. After correcting the distance effect, the field and laboratory measurements agree quite well. In Fig. 12, these two are compared with the incidence angle function of a 30\% reflecting tarp, a target with approximately similar brightness to that of Kivenlahti sand. The results are similar to those obtained for Spectralon in Section 3.3, which points out that laboratory-derived results could be used to correct field measured TLS after the distance effect has been corrected. The sand data appears more scattered than that measured for the Spectralon plate. This is often the case with 'real-world' data, but it might also indicate a minor effect from the surface macro topography or roughness, similar to that of the Assuan sand (Fig. 2).

\section{CONCLUSIONS}

The results in this paper show that the intensity distance effect and incidence angle effect do not mix, which makes it possible to correct both by using different models that are independent of the measurement. We also compared laboratory-measured intensity values with field scans, and our first results suggest that the incidence angle effect can be corrected with correction functions that depend on the object brightness.

We investigated the effect of object grain size to the incidence angle effect with a variety of different grain size sand and sugar samples. Our results show that variations on the surface topography that are smaller than the laser spot on the target do not have a significant effect on the incidence angle effect. The effect of surface topography should be studied further with a larger variation of target surface roughness than that available for this study. However, the samples studied in this paper represent a typical range of targets available in, e.g., urban remote sensing (e.g., walls, roads, roofs, etc.).

The first results from the hyperspectral Lidar experiment indicate that the incidence angle effect does not depend on the scanning wavelength, which means that the same correction models can be used for different scanners regardless of the wavelength of the scanner. This finding must be further assessed in future hyperspectral experiments, and with a wide selection of targets with different spectral propreties., The first results suggest that other effects than the wavelength are more significant in the incidence angle correction of TLS intensity data. 


\section{ACKNOWLEDGEMENT}

This research was funded by the Academy of Finland research projects "New techniques in active remote sensing: hyperspectral laser in environmental change detection" and "Mobile hyperspectral laser remote sensing".

The authors would like to thank Antero Kukko's support in the measurements and Finnsugar Ltd for sugar information.

\section{REFERENCES}

Ahokas, E.; Kaasalainen, S.; Hyyppä, J.; Suomalainen, J. 2006. "Calibration of the Optech ALTM 3100 laser scanner intensity data using brightness targets". Int. Arch. Photogramm. Remote Sens. Spat. Inf. Sci., 34, pp. 3-6.,

Coren, F.; Sterzai, P. 2006. "Radiometric correction in laser scanning”. Int. J. Remote Sens., 27, pp. 3097-3104,

Hakala, T., Suomalainen, J., Kaasalainen, S., and Chen, Y. 2012. "Full Waveform Hyperspectral LiDAR for Terrestrial Laser Scanning”. Optics Express, 20(7), 7119-7127.

Hollaus M., Mandlburger G., Pfeifer N., Mücke W., 2010. Land cover dependent derivation of digital surface models from airborne laser scanning data. In: ISPRS Archives. PCV 2010, Paris, France, Vol. 39(3). pp. 6,

Höfle, B., Hollaus, M., Hagenauer, J. "Urban vegetation detection using radiometrically calibrated small-footprint fullwaveform airborne LiDAR data". ISPRS J. Photogramm. Remote Sens. Volume 67, pp. 134-147, (2012).

Höfle, B.; Pfeifer, N. "Correction of laser scanning intensity data: Data and model-driven approaches". ISPRS J. Photogramm. Remote Sens., 62, pp. 415-433, (2007).

Jutzi, B.; Gross, H. "Normalization of LiDAR intensity data based on range and surface incidence angle". Int. Arch. Photogramm. Remote Sens. Spat. Inf. Sci. 2009, 38, 213-218, (2009).

Kaasalainen, S., Jaakkola, A., Kaasalainen, M., Krooks, A., and Kukko, A. 2011. "Analysis of incidence angle and distance effects on terrestrial laser scanner intensity: Search for correction methods". Remote Sensing, 3(10), pp. 2207-2221.

Kaasalainen, S.; Krooks, A.; Kukko, A.; Kaartinen, H. 2009a. "Radiometric calibration of terrestrial laser scanners with external reference targets". Remote Sensing, 1, 144-158.

Kaasalainen, S.; Hyyppä, H.; Kukko, A.; Litkey, P.; Ahokas, E.; Hyyppä, J.; Lehner, H.; Jaakkola, A.; Suomalainen, J.; Akujärvi, A. 2009b. "Radiometric calibration of LIDAR intensity with commercially available reference targets". IEEE Trans. Geosci. Remote Sens., 47, pp. 588-598.

Kukko, A.; Kaasalainen, S.; Litkey, P. 2008. "Effect of incidence angle on laser scanner intensity and surface data". Appl. Opt., 47, 986-992.

Lehner, H.; Briese, C. 2010. Radiometric Calibration of FullWaveform Airborne Laser Scanning Data Based on Natural Surfaces. In Proceedings of ISPRS Technical Commission VII
Symposium, 100 Years ISPRS, Advancing Remote Sensing Science, Vienna, Austria, 5-7 July 2010; In IAPRS; 2010; Volume 38, pp. 360-365.

Lichti, D.D. 2005. "Spectral filtering and classification of terrestrial laser scanner point clouds". Photogramm. Rec., 20, 218-240.

Lichti, D.D. 2003. "Modelling of Laser Scanner NIR Intensity for Multi-Spectral Point Cloud Classification". In Proceedings of Optical 3D Measurement Techniques VI, Zürich, Switzerland, pp. 282-289.

Pesci, A.; Teza, G. 2008. "Effects of surface irregularities on intensity data from laser scanning: An experimental approach". Ann. Geophys., 51, 839-848.

Pfeifer, N., Höfle, B., Briese, C., Rutzinger, M., Haring, A. 2008. "Analysis of the backscattered energy in terrestrial laser scanning data". In: ISRPS Archives,Vol. XXXVII, Part B5, Beijing, 2008, pp. 1045-1052.

Soudarissanane, S., Lindenbergh, R., Menenti, M. and Teunissen P. 2011. "Scanning geometry: Influencing factor on the quality of terrestrial laser scanning points". ISPRS Journal of Photogr. and RS., Volume 66, Issue 4, p. 11.

Voegtle, T.; Wakaluk, S. 2009. "Effects on the Measurements of the Terrestrial Laser Scanner HDS 6000 (Leica) Caused by Different Object Materials". In Proceedings of Laserscanning 2009, Paris, France, 1-2 September 2009; In IAPRS, Volume 38, pp. 68-74.

Wagner, W.; Ullrich, A.; Ducic, V.; Melzer, T.; Studnicka, N. "Gaussian decomposition and calibration of a novel smallfootprint full-waveform digitising airborne laser scanner". ISPRS J. Photogramm. Remote Sens., 60, pp. 100-112, (2006).

Yan, W.Y., Shaker, A., Habib, A., Kersting A.P. 2012. "Improving classification accuracy of airborne LiDAR intensity data by geometric calibration and radiometric correction", ISPRS J. Photogramm. Remote Sens, Volume 67, pp 35-44.

Pfeifer, N., Höfle, B., Briese, C., Rutzinger, M., Haring, A. 2008. "Analysis of the backscattered energy in terrestrial laser scanning data". In: Int. Arch. Photogramm. Remote Sens. Spat. Inf. Sci, Vol. XXXVII, Part B5, Beijing, 2008, pp. 1045-1052. 